Rapid Reviews COVID-19

\title{
Review 2: "Optimal SARS- \\ CoV-2 vaccine allocation \\ using real-time \\ seroprevalence estimates \\ in Rhode Island and \\ Massachusetts"
}

\section{François Castonguay ${ }^{1}$}

${ }^{1}$ University of California Davis, Agricultural and Resource Economics, United States

Published on: Mar 01, 2021

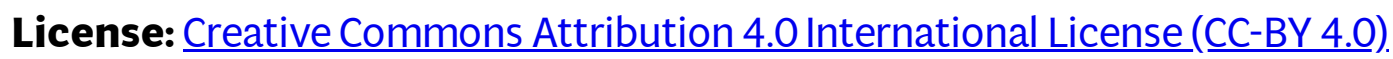




\section{$\underline{\text { RR:C19 Evidence Scale rating by reviewer: }}$}

- Reliable. The main study claims are generally justified by its methods and data. The results and conclusions are likely to be similar to the hypothetical ideal study. There are some minor caveats or limitations, but they would/do not change the major claims of the study. The study provides sufficient strength of evidence on its own that its main claims should be considered actionable, with some room for future revision.

$* * * * * * * * * * * * * * * * * * * * * * * * * * * * * * * * * * * * * * *$

\section{Review:}

The word "optimal" is inappropriate in this context. The methodology used by the authors does not allow them to claim that the strategy they propose is optimal. When the authors talk about optimality, what they in fact mean is "the best among the strategies evaluated" (as the authors correctly point out on Page 7). This method is called scenario analysis, in which several ad hoc scenarios are compared with each other, and the one that has e.g. the lowest number of deaths is the best scenario. The reasoning here is that the authors only consider a subset of all potential strategies and hence cannot claim that the one they propose is optimal. For instance, it possible that sharing vaccines between groups in another way than the ones considered in the manuscript (e.g. 75/25, 50/50, 25/75) is better, or that splitting up the age groups in different segments would be better. Hence, the word "optimal" is misleading and I recommend avoiding using this word, especially in the title of the manuscript, and even in the context of "sub-optimal," because technically all proposed strategies in the manuscript could be sub-optimal and there is no way of currently verifying that.

Another concern I have is the authors' claim that "[i]n our model, groups that are at high risk of death if infected are the older age groups, but our analysis implies that any high-risk group-whether the risk factor is age, obesity, diabetes, past lung disease, lack of health care access, or anything else-should have an equally high priority to vaccination." It seems possible that the other factors that increase the risk of death if infected are correlated with a variable that the current risk factor they consider (i.e. age) is not correlated with. As a result, this other risk factor may not require as high vaccination priority as the older high-risk population. For instance, individuals with past lung diseases may have hands-on experience of what needing a ventilator to 
breathe feels like and as such, these individuals may have a more risk-averse behavior which in turn implies that they are less exposed to SARS-CoV-2 than older individuals. For this reason, I believe this claim is misleading, and fully addressing this question would require further analyses.

I believe this manuscript is important as it highlights the importance of having information on seroprevalence. However, I do not think the authors do a good job at presenting this result (it is merely presented as a secondary result), and there lacks an explanation on how their work differs from Bubar et al. [14]'s work. Finally, I would like to see the authors detail more their model in the supplementary material. It is very helpful for some readers to be able to see the state equations. 